\title{
Data quality of Aeolus wind measurements
}

\section{Isabell Krisch ${ }^{1}$}

Christian Lemmerz ${ }^{1}$, Oliver Lux ${ }^{1}$, Uwe Marksteiner ${ }^{1}$, Nafiseh Masoumzadeh ${ }^{1}$, Oliver Reitebuch ${ }^{1}$, Fabian Weiler ${ }^{1}$, Benjamin Witschas ${ }^{1}$, Fabio Bracci ${ }^{2}$, Markus Meringer ${ }^{2}$, Karsten Schmidt ${ }^{2}$, Alexander Geiß ${ }^{3}$, Dorit Huber ${ }^{4}$, Ines Nikolaus ${ }^{5}$, Michael Vaughan ${ }^{6}$, Alain Dabas ${ }^{7}$, Thomas Flament ${ }^{7}$, Dimitri Trapon ${ }^{7}$, Saleh Abdalla ${ }^{8}$, Lars Isaksen ${ }^{8}$, Michael Rennie ${ }^{8}$, Dave Donovan ${ }^{9}$, Jos de Kloe ${ }^{9}$, Gert-Jan Marseille ${ }^{9}$, Ad Stoffelen ${ }^{9}$, Thomas Kanitz ${ }^{10}$, Anne-Grete Straume ${ }^{10}$, Denny Wernham ${ }^{10}$, Jonas von Bismarck ${ }^{11}$, Sebastian Bley ${ }^{11}$, Peggy Fischer ${ }^{11}$, Tommaso Parrinello ${ }^{11}$

${ }^{1} D L R$, Institute of Atmospheric Physics ${ }^{2} D L R$, Remote Sensing Technology Institute ${ }^{3}$ Ludwig-Maximilians-Universität München ${ }^{4}$ DoRIT

${ }^{5}$ Munich University of Applied Sciences ${ }^{6}$ OLA

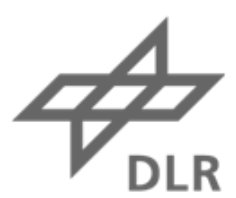

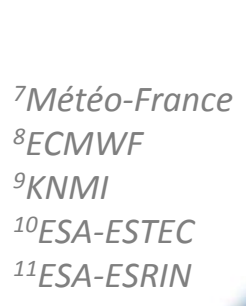

${ }^{7}$ Météo-France

${ }^{8} \mathrm{ECMWF}$

${ }^{9} \mathrm{KNMI}$

${ }^{11}$ ESA-ESRIN

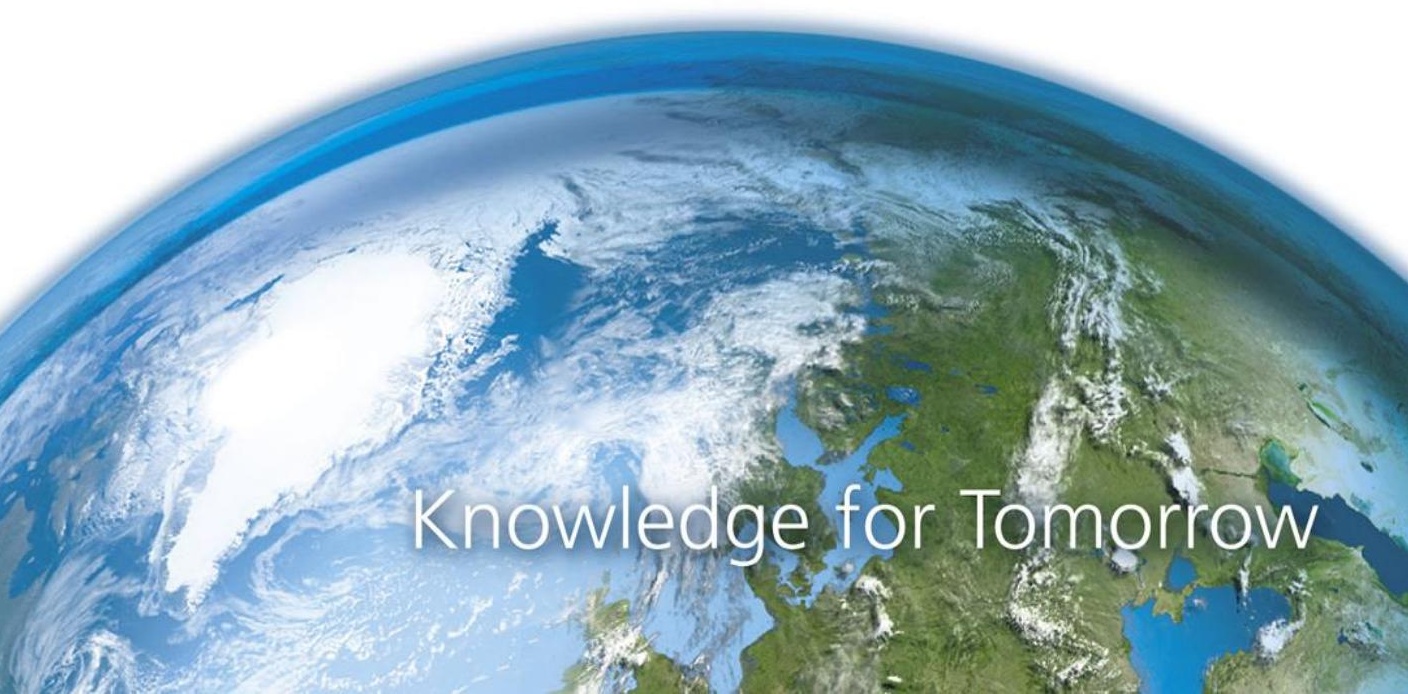




\section{The Aeolus Data Innovation and Science Cluster (DISC)}

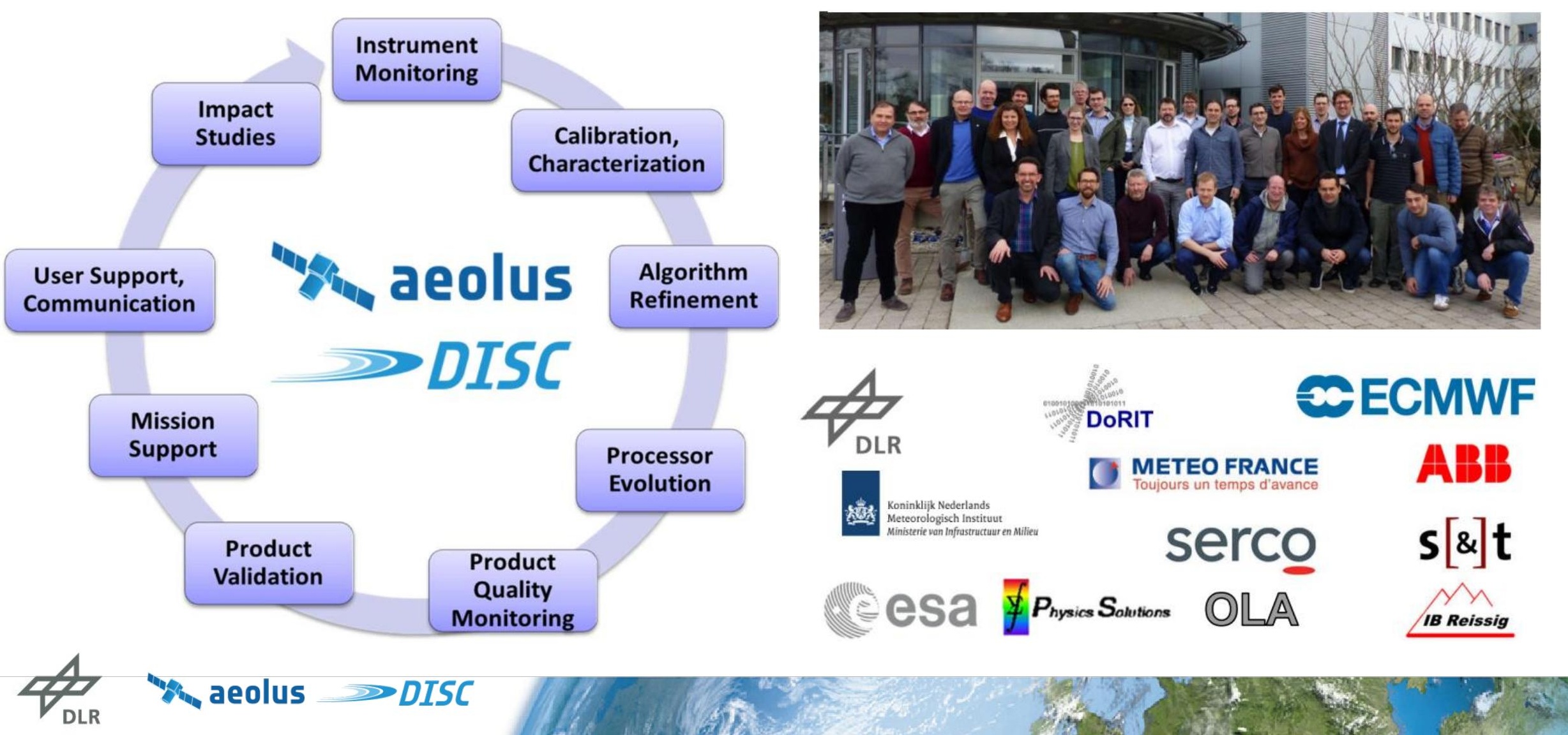




\section{O-B:}

Difference between Aeolus observation and ECMWF forecasted HLOS wind

(C) M. Rennie (ECMWF) All rights reserved.

\section{ECMWF operational monitoring of Aeolus Rayleigh and Mie winds}

Mie cloudy global, daily O-B statistics
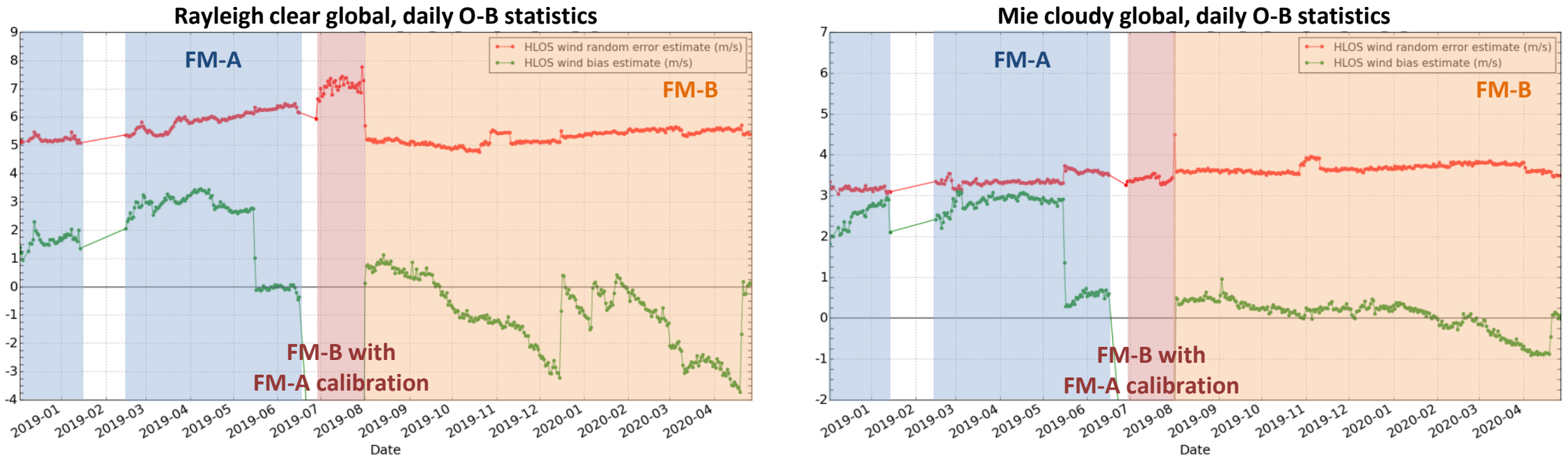

- Random error of ALADIN Rayleigh winds is in the order of 5-7 m/s and 3-4 m/s for Mie winds (mostly clouds): random errors in both channels were increased since launch and did not improve.

- Systematic errors (bias) for both Mie and Rayleigh winds were enhanced since launch (several $\mathrm{m} / \mathrm{s}$ ), and show strong temporal variations (slow drifts), orbital variations, differences for ascending and descending orbits, and occurrence in some range-gates $>$ combination of 4 unexpected sources for the bias identified up to now 


\section{Evolution of Aeolus random and systematic errors}

Comparison of Aeolus Rayleigh and Mie winds with 4 German radar wind-profilers
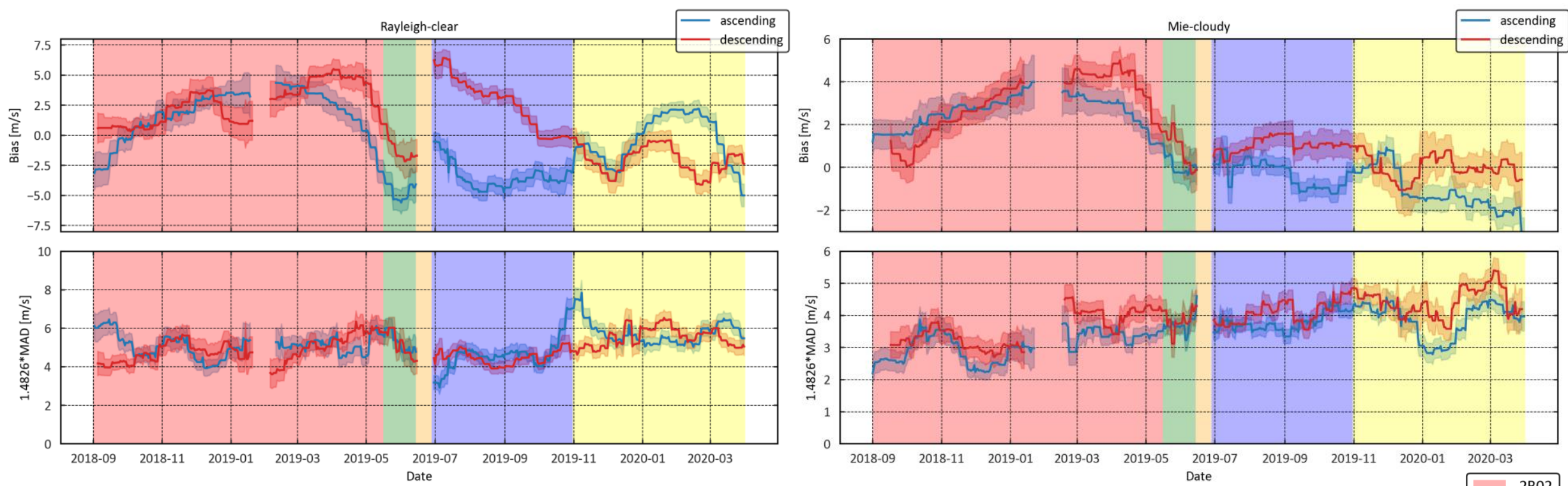

- $\quad$ Radar wind profilers support error estimates obtained by ECMWF O-B.

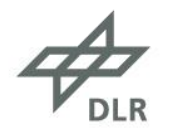

$\checkmark n$ aeolus $\gg D I S C$

(C) A. Geiß (University LMU Munich). All rights reserved. 


\section{What drives the random errors?}

1. Laser emit energy

$\Rightarrow$ Lower than expected $(60 \mathrm{~mJ}$ instead of $80 \mathrm{~mJ})$

$\Rightarrow$ Negative trend

2. Optical signal throughput in receive path for atmospheric signal

$\Rightarrow$ Lower than expected (factor 2-3)

$\Rightarrow$ Negative trend

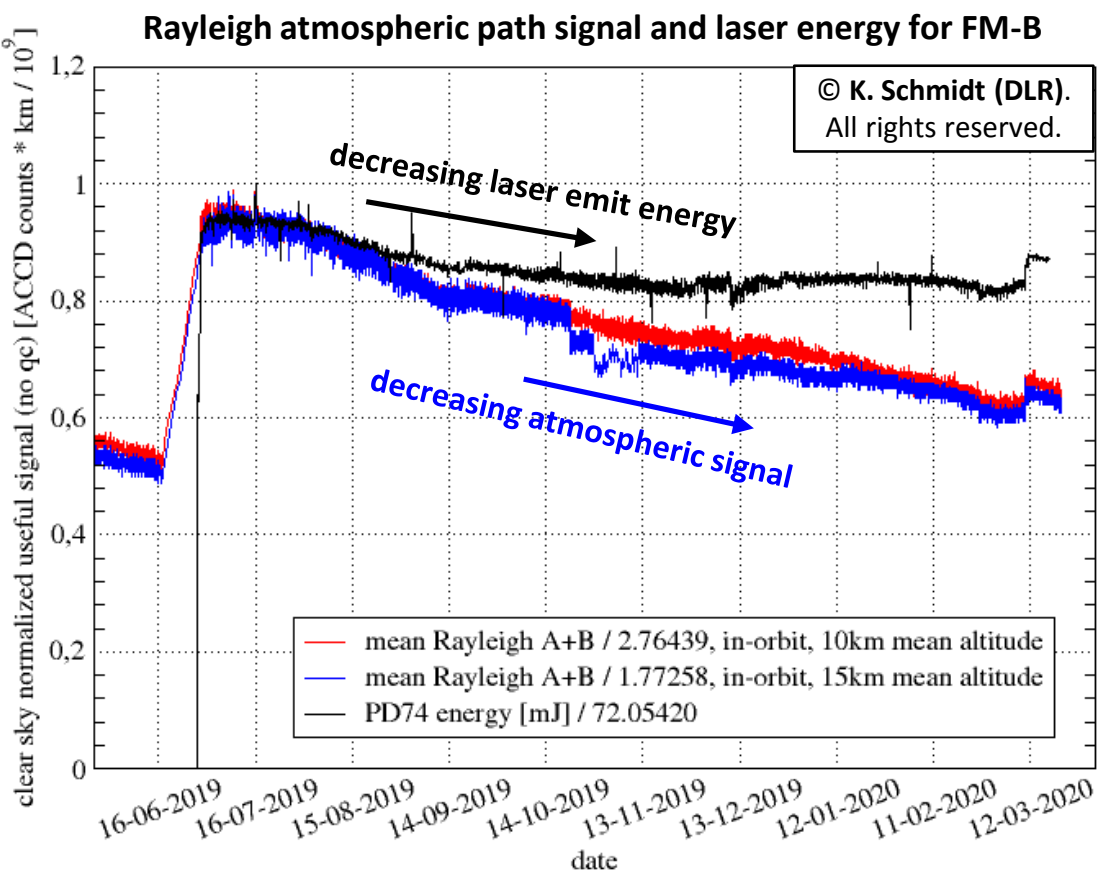

Discrepancy between these lines indicates that laser energy is not representative for instrument performance. This hints to a signal loss in optical emit and/or receive path. 
Orbital variation of Rayleigh solar background noise

\section{What drives the random errors?}

1. Laser emit energy

$\Rightarrow$ Lower than expected (factor 1-2)

$\Rightarrow$ Negative trend

2. Optical signal throughput in receive path for atmospheric signal

$\Rightarrow$ Lower than expected (factor 2-3)

$\Rightarrow$ Negative trend

3. Solar background noise

$\Rightarrow$ Impact higher than expected due to lower atmospheric signal

$\Rightarrow$ Seasonal variation of solar background by factor 18: Rayleigh random errors of 7-8 m/s were obtained in summer months for polar regions
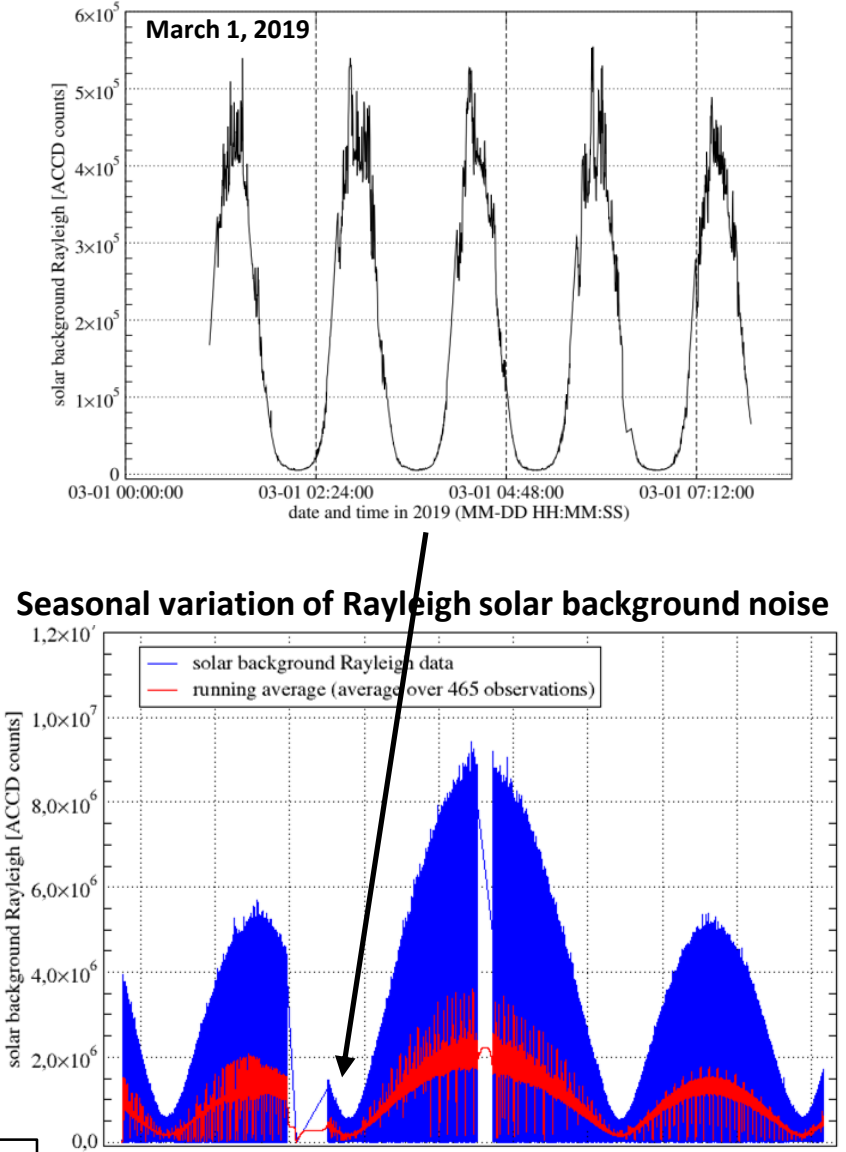

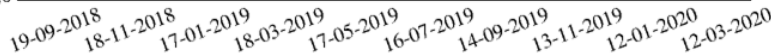




\section{What causes systematic errors?}

\section{Combination of 4 unexpected error sources with}

different temporal characteristics

1. Higher dark current rates for some "hot pixels"

$\Rightarrow$ affects specific range gates; currently 13 pixels on Mie ACCD and 14 pixels on Rayleigh ACCD

April 2020, Hot pixel map
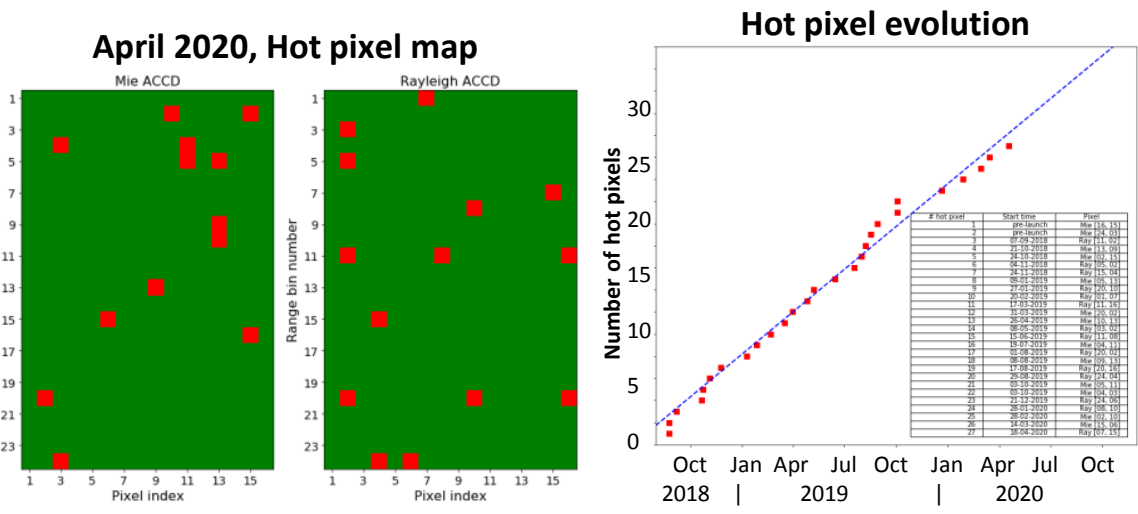

Hot pixel evolution
Systematic dark signal offsets with $10^{-3}$ to $10^{-4}$ of signal or $1 \%-10 \%$ of noise

Hot pixels (mean per DCMZ/DUDE measurement)
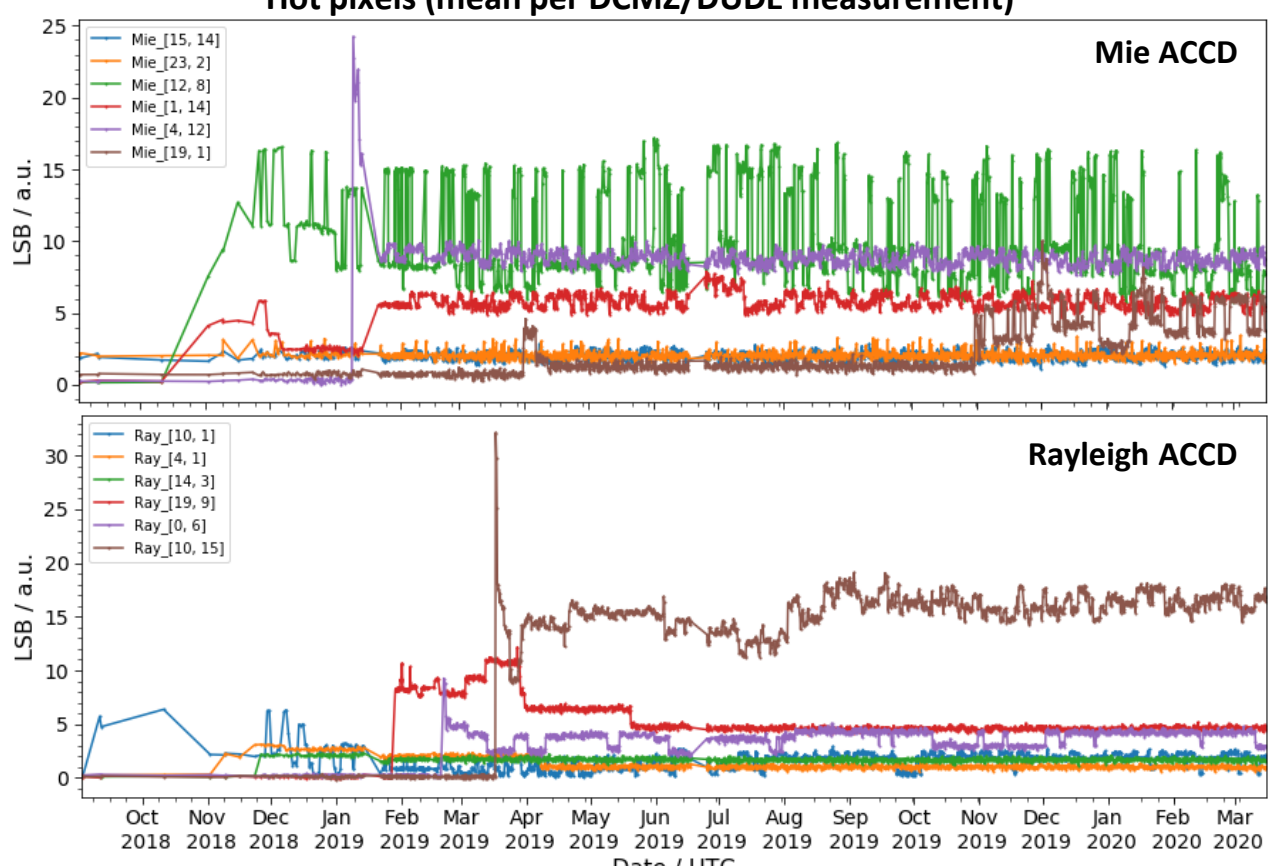
Date / UTC

(C) F. Weiler (DLR). All rights reserved.

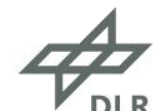




\section{Correction of hot pixels implemented in June 2019}

\begin{tabular}{|c|}
\hline C Fig: VirES for Aeolus; \\
correction developed by \\
F. Weiler (DLR) and D. Huber (DoRIT) \\
All rights reserved. \\
\hline
\end{tabular}

Enhanced dark signals (hot pixels) cause systematic errors of $\mathbf{\pm}(\mathbf{1 - 3} \mathrm{m} / \mathrm{s})$ for Rayleigh winds for some range gates => horizontal stripes

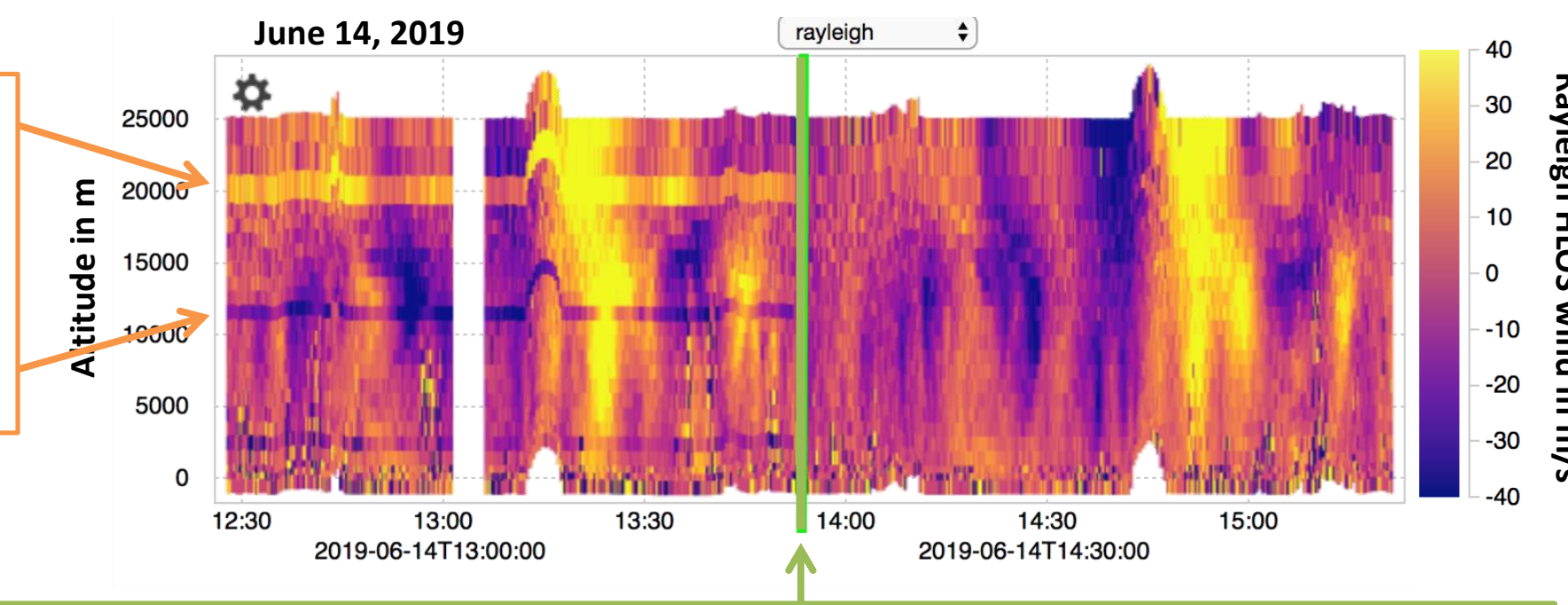

New instrument modes introduced, algorithms developed and implemented in operational processors on June, 14, 2019 for correction of hot pixels by measuring dark signals 4 times per day for real-time datasets

Hot pixels appearing in between the dark signal measurements still cause biases in NRT dataset (are flagged in L2B winds) 


\section{What causes systematic errors?}

\section{Combination of 4 unexpected error sources with} different temporal characteristics

1. Higher dark current rates for some "hot pixels"

2. Error in the on-board software in calculation of residual projection of the satellite ground speed on the line-of-sight LOS:

$\Rightarrow$ harmonic variation of bias along the orbit

$\Rightarrow$ correction with on-board calculated $v_{S A T}$ in L1B and L2B processors de-activated in summer 2019

$\Rightarrow$ correction for on-ground L1A processing identified and implementation envisaged for autumn 2020
$\mathrm{v}_{\mathrm{SAT}}$ during Dec $18-24,2018$
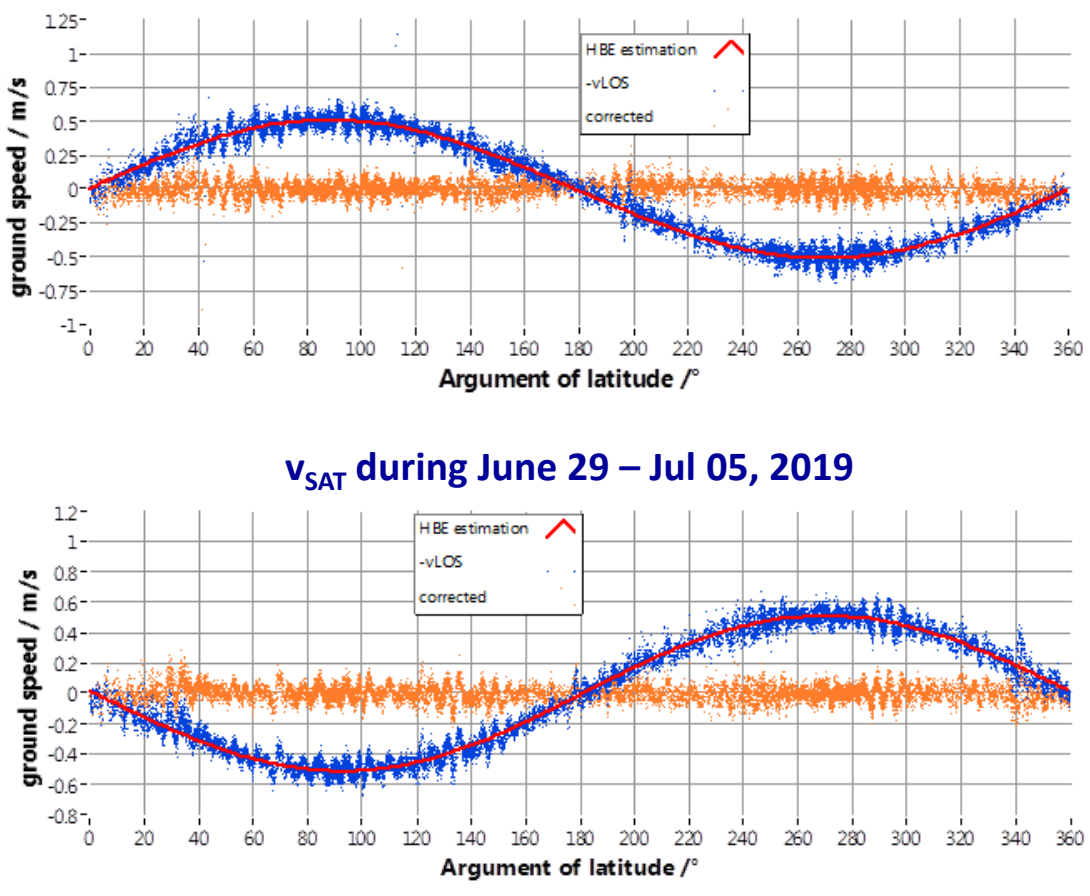

Amplitude of $\mathrm{v}_{\mathrm{LOS}}$ is zero at equator and maximum at poles with around $0.6 \mathrm{~m} / \mathrm{s}$, but opposite phase as in December 


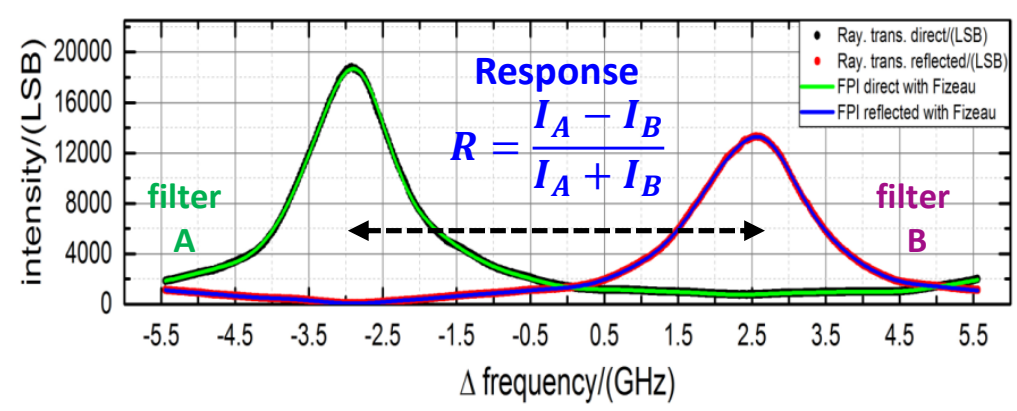

1. Higher dark current rates for some "hot pixels"

2. Error in the on-board software in calculation of residual projection of the satellite ground speed on the line-of-sight LOS

3. Slow drifts in the illumination of the Rayleigh/Mie spectrometers causing a slowly, linear drifting constant bias

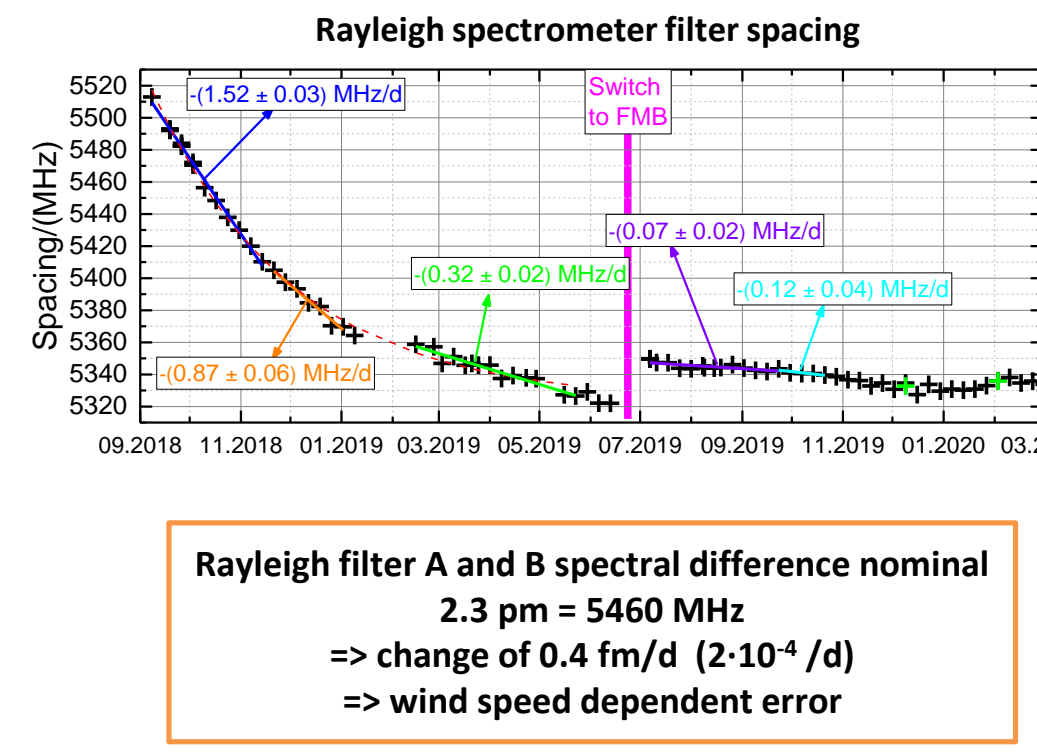




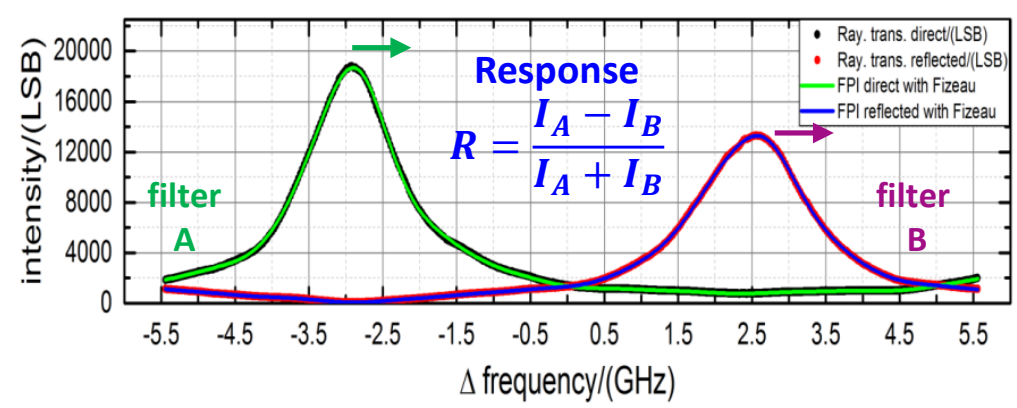

1. Higher dark current rates for some "hot pixels"

2. Error in the on-board software in calculation of residual projection of the satellite ground speed on the line-of-sight LOS

3. Slow drifts in the illumination of the Rayleigh/Mie spectrometers causing a slowly, linear drifting constant bias
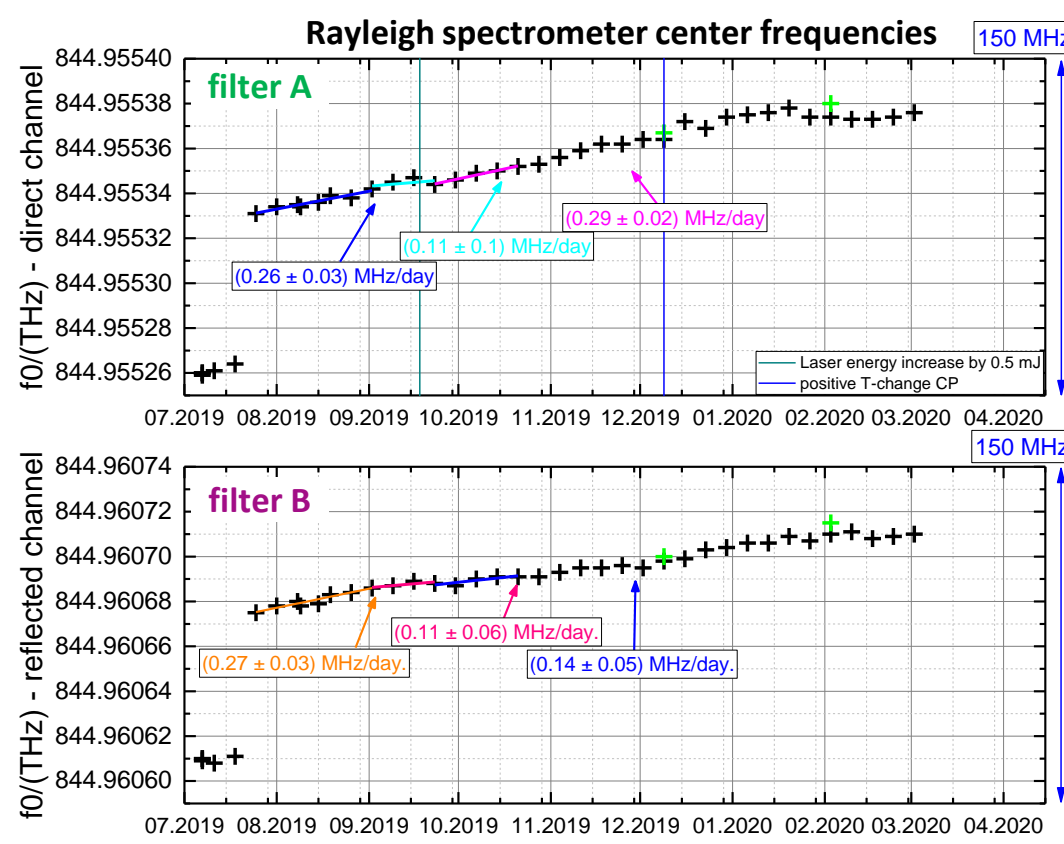


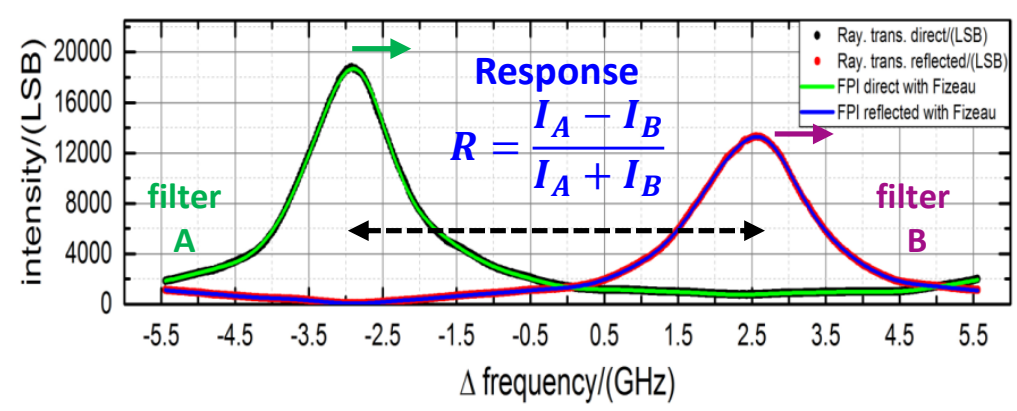

1. Higher dark current rates for some "hot pixels"

2. Error in the on-board software in calculation of residual projection of the satellite ground speed on the line-of-sight LOS

3. Slow drifts in the illumination of the Rayleigh/Mie spectrometers causing a slowly, linear drifting constant bias

$\Rightarrow$ Was in the past corrected manually by tuning of input parameters for $\mathrm{L} 2 \mathrm{~B}$ processing

$\Rightarrow$ Now corrected in real-time products as part of M1-correction (see next slide)

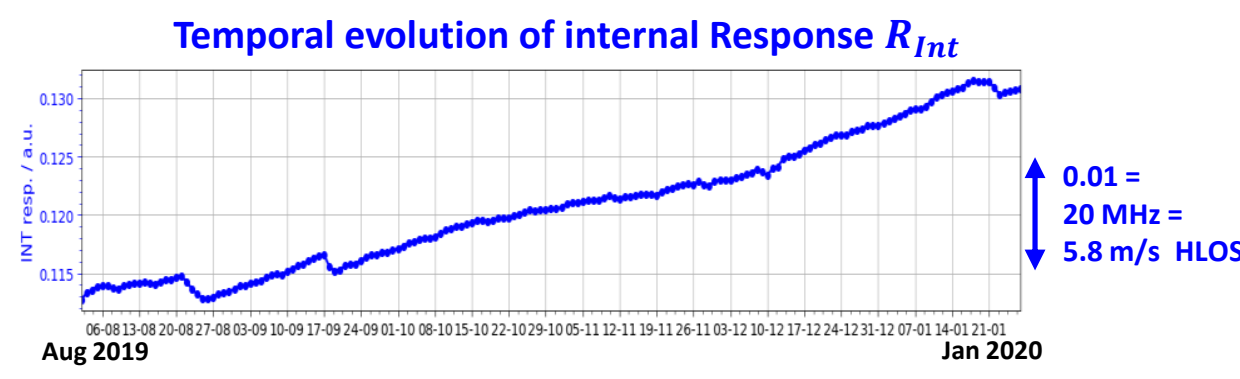




\section{What causes systematic errors?}

Combination of 4 unexpected error sources with different temporal characteristics

1. Higher dark current rates for some "hot pixels"

2. Error in the on-board software in calculation of residual projection of the satellite ground speed on the line-of-sight LOS

3. Slow drifts in the illumination of the Rayleigh/Mie spectrometers causing a slowly, linear drifting constant bias

4. Thermal variations of the $\mathbf{M} \mathbf{1}$ telescope mirror

$\Rightarrow$ Rayleigh bias with orbital phase (argument of latitude) and longitude

$\Rightarrow$ Use correlation between M1 temperatures and mean model bias for correction
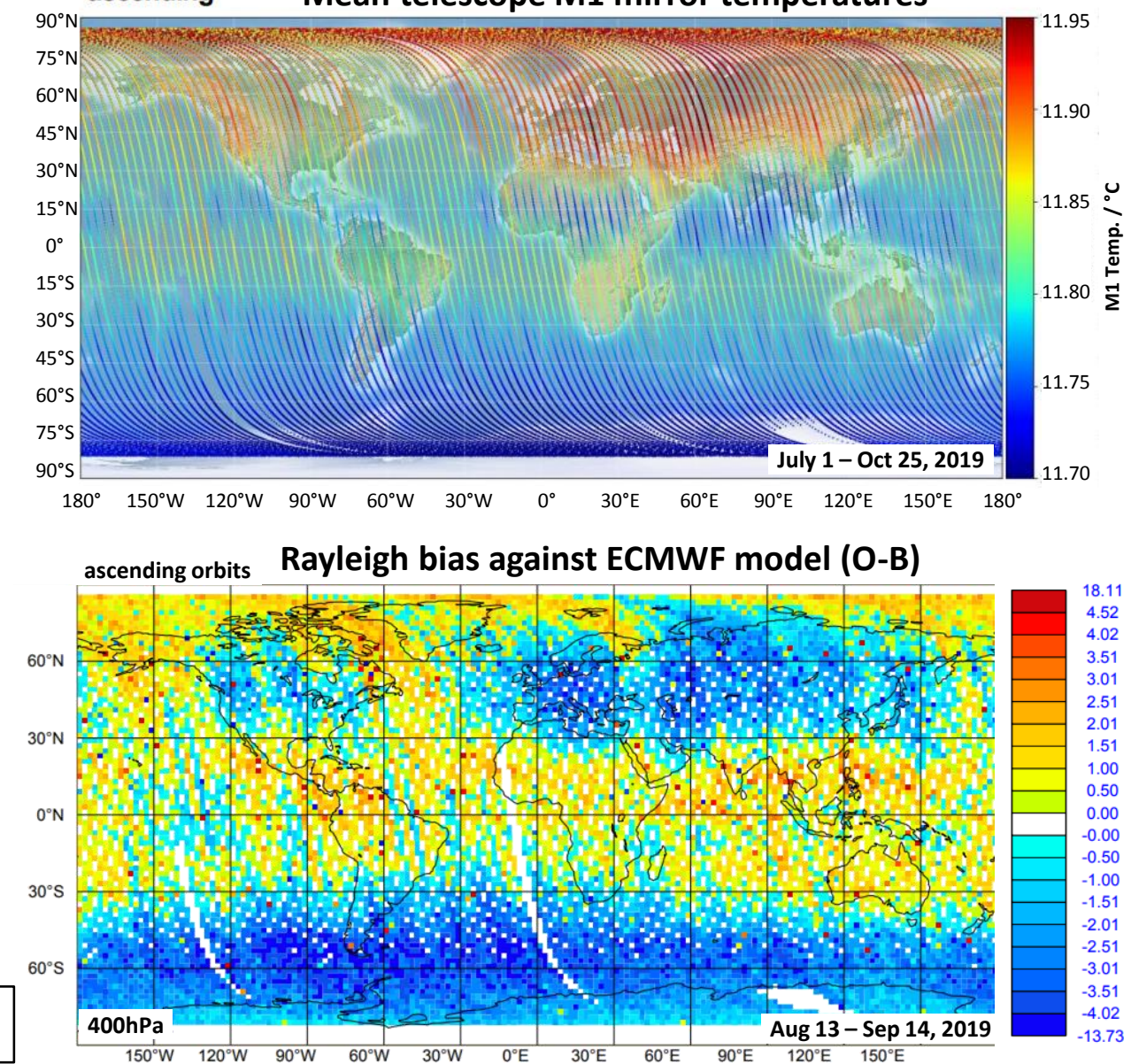

"su, aeolus $\gg$ DISC




\section{Correction of bias caused by thermal variations of the M1 mirror}

\section{Multiple linear regression (1d of data)}

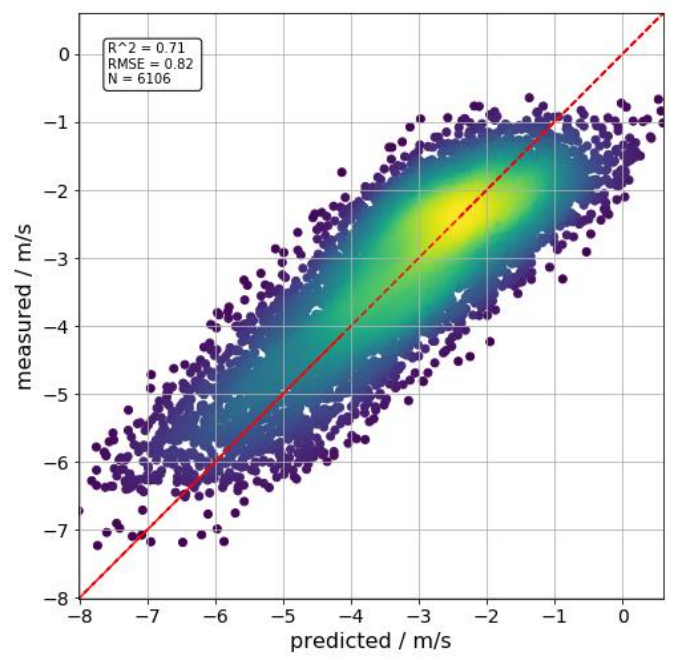

Rayleigh bias versus time on 09/08/2019

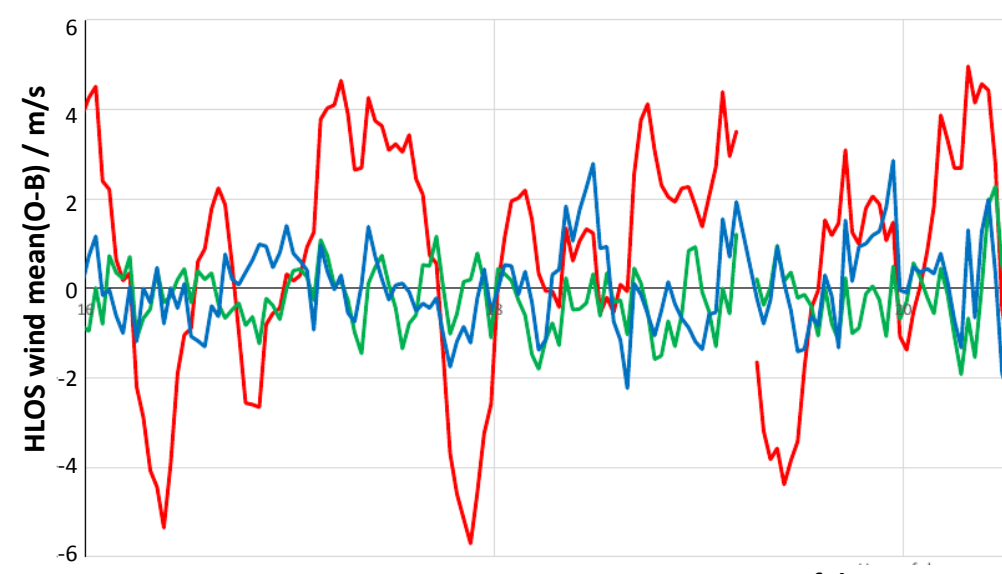

Hour of day

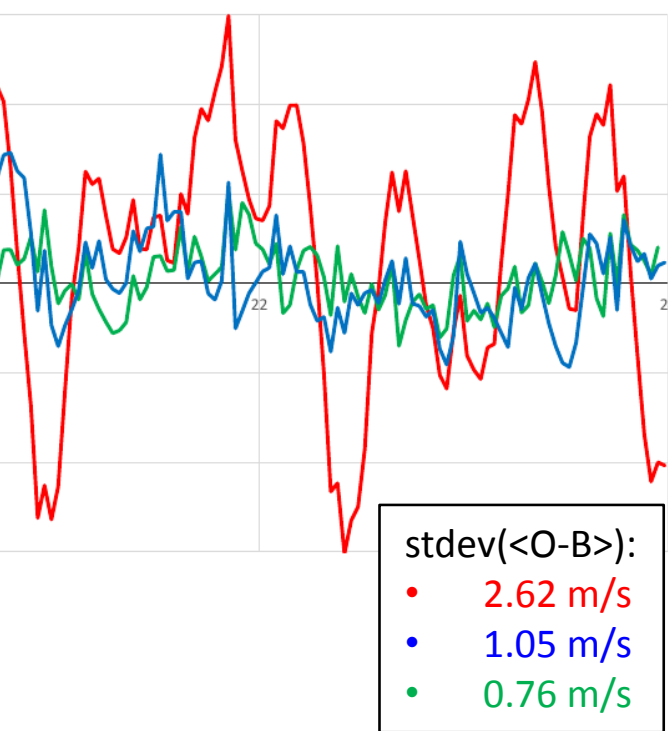

\section{Effects of M1 correction:}

1. „flattens out" orbital variation (M1 telescope mirror) - reduces std. deviation of O-B (here: $2.62 \mathrm{~m} / \mathrm{s}$ to $0.76 \mathrm{~m} / \mathrm{s}$ )

2. Corrects for bias drifts (e.g. illumination effects) 


\section{Correction of bias caused by thermal variations of the M1 mirror}

\section{Activated on April 20, 2020}
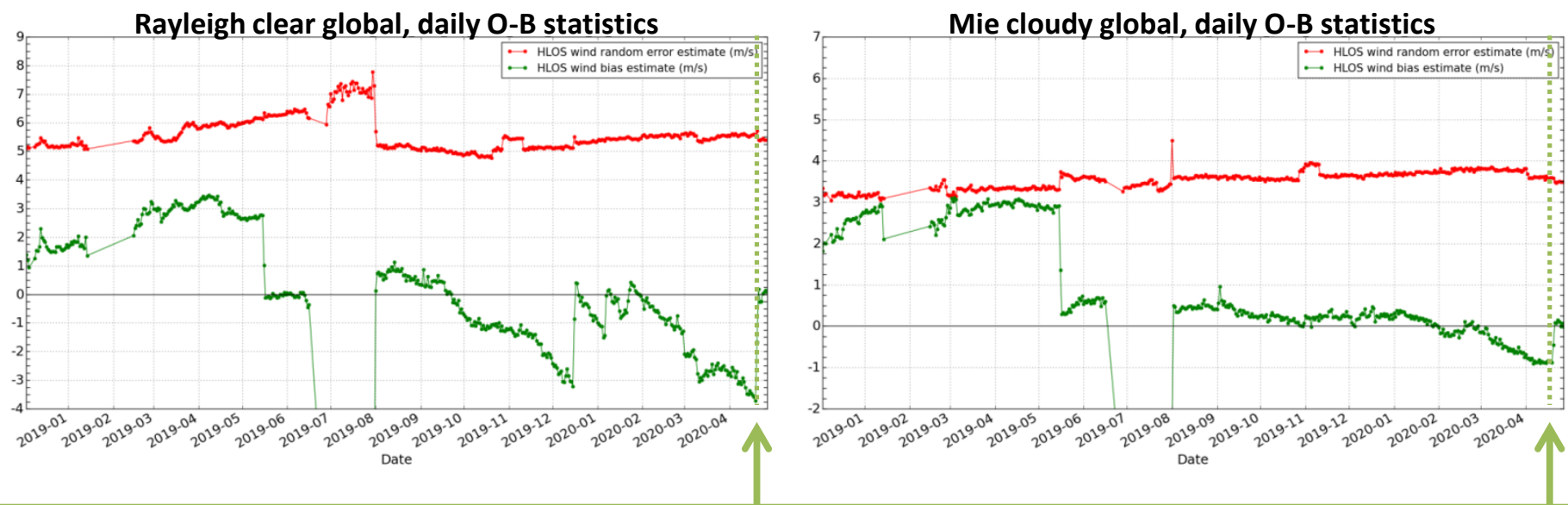

New instrument modes introduced, algorithms developed and implemented in operational processors on April, 20, 2020 for correction of M1 temperature biases and slowly, linear drifting constant biases by using daily mean correlation between ECMWF model bias (O-B) and M1 temperature measurements.

Additional investigations are on-going to use ground measurements instead of ECMWF O-B to regain model independence. 


\section{Re-processing of Aeolus data}

- Re-processing activities started beginning of this year

- It involves multiple manual and only semi-automated steps, e.g.

- Manual production of calibration files and processing up to $\mathrm{L} 1 \mathrm{~B}$

- Correction of hot pixels also in time periods between dark signal measurements

- Semi-automated processing up to L2B

- Manual estimation of M1 temperature correction

- Verification of bias correction and delivery of all calibration files to ESA

- Re-processing at ESA

- Validation and quality control of re-processed dataset

- $\quad$ First re-processed dataset is FM-B data from 2019.

It will be available in Sept. 2020.

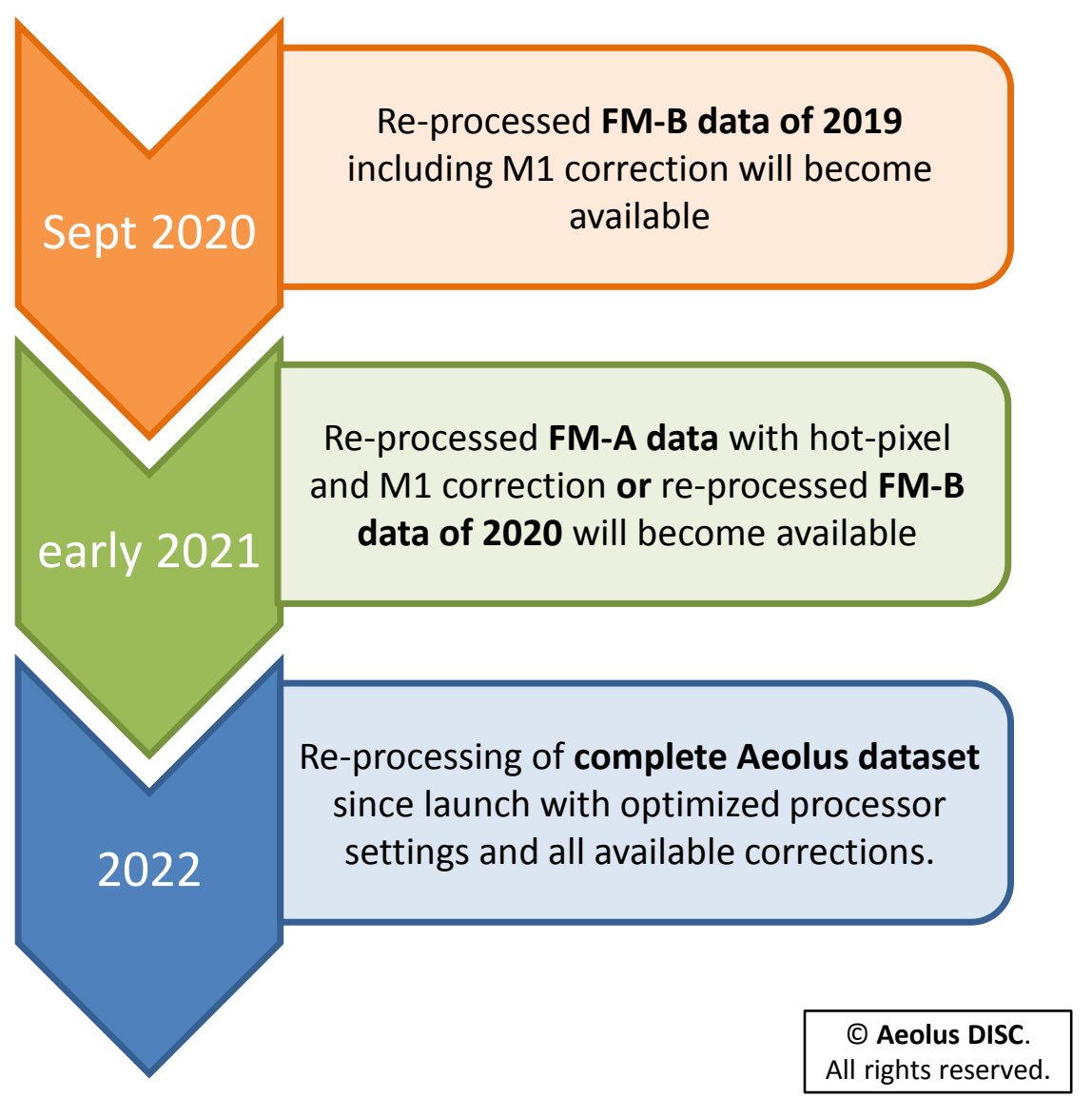




\section{Summary and Conclusion}

- The Aeolus DISC consortium is responsible for calibration, processor evolution, product quality and impact studies.

- Both systematic and random errors after launch were higher than expected

- Precise instrument characterization and use of O-B statistics enabled a drastic reduction of systematic errors

- Hot pixel correction was implemented in June 2019 for NRT data stream

- M1 temp. correction was implemented in April 2020 for NRT data stream and is currently examined by experts.

> Public data release on May 12, 2020 (:)

- Re-processing started recently and first re-processed data (June - December 2019) will be available in Sept. 2020.

\section{Maeolus $\gg D I S C$}

Hot pixel correction implemented

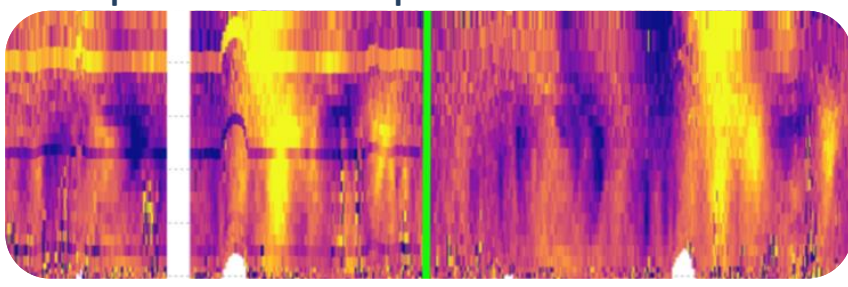

M1 bias correction implemented

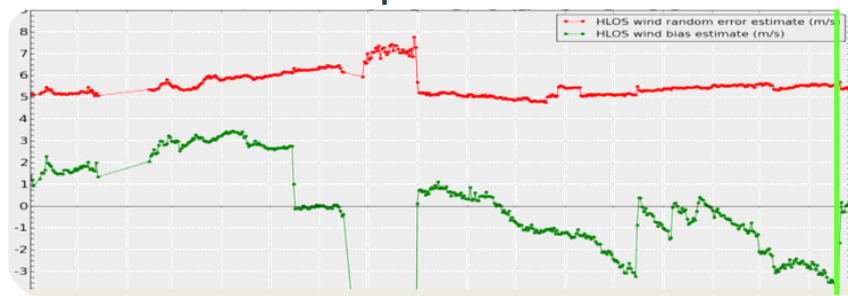

Public NRT data release

12 May 2020

Reprocessing

\begin{tabular}{|c|c|c|}
\hline Sept. 2020 & early 20 & 2022 \\
\hline FM-B 2019 data & FM-A data & $\begin{array}{c}\text { Complete } \\
\text { Aeolus dataset }\end{array}$ \\
\hline
\end{tabular}

